\title{
Incorporating Deblurring Techniques in Multiple Recognition of License Plates from Video Sequences
}

\author{
Ria Ambrocio Sagum, MCS ${ }^{a}$, Anthony John G. Gilo ${ }^{b}$ and Mary Ann D. Narag \\ ${ }^{a}$ Department of Computer Science, College of Computer and Information SciencesResearch Management Office \\ ${ }^{b, c}$ Polytechnic University of the Philippines, Sta. Mesa, Manila \\ Email: ${ }^{\mathrm{a}}$ rasagum@pup.edu.ph, ${ }^{\mathrm{b}}$ anthonygilo.10@gmail.com, ${ }^{\mathrm{c}}$ meannarag@gmail.com
}

Article History: Received: 10 November 2020; Revised 12 January 2021 Accepted: 27 January 2021; Published online: 5 April 2021

\begin{abstract}
Recognition of license plate is the process of wherein photographic video or images of license plates are being captured and then processed using an application that implements series of algorithms that will provide the alpha numeric conversion of the captured data. In this study, the researchers developed a license plate recognition that incorporates image deblurring to accommodate multiple recognition from video sequences. The approach uses Background Subtraction and Connected Component Analysis for the detection of license plates, Image deblurring to enhance the image and reduce the difficulties in recognition, and LBP Cascade Classifier was implemented for recognition of characters. Since multiple detection for license plate produces different difficulties such as motion blur and camera angle view the approach attempts to minimize the effects of these problems while still being applicable to multiple detection. 30 videos with 3 minutes length each of actual traffic situation were gathered and recorded at the footbridge of UP Ayala Technohub, Commowealth Ave. Quezon City, Philippines and 10 of these videos were used as input for the testing and experiment of the system. The accuracy for plate detection were computed using F-measure which yields to $87.32 \%$ for both system with image deblurring and none, while the accuracy for character recognition is $62.66 \%$ for system with image deblurring and $48.25 \%$ for the system without image deblurring. The result shows that there is a significant difference in the accuracy of license plate recognition between the system with image deblurring and without image deblurring.
\end{abstract}

Keywords: Character Recognition, Image Deblurring, License Detection, LPR, LBP Cascade Classifier

\section{Introduction}

Automated license plate recognition (alpr) is known technology that automatically reads car plates and also provides an automated way to detect vehicles that committed a license plate related violation [1]. There are variety of ways that alpr systems can be deployed, this includes mobile alpr which is mounted in vehicle cameras and fixed or portable alpr that are affixed permanently to a certain structure like bridge, light pole or overhead signs [2]. However, existing systems are developed that take considerations of different style, format, and character sets for license plates and is therefore not capable of accommodating different formats like the one that is being used in the philippines [3]. In addition, there are number of challenges as far as mathematical aspects of alpr frameworks that is not yet precisely determined and is necessary to design tough mathematical model to address such issues.

Vehicle image quality is sometimes poor because of blurring effects. There are distinctive reasons for this, such as the movement of camera, out-of-focus or camera is too far away from the object. The degraded vehicle images by blurring effects decrease the accuracy of alpr4].

Acquisition phase is critical in such a way that unconstrained environment for example outdoor scenes has a tendency to produce not so good lighting and contrast due to overexposure, reflection or shadows for this reason, proper illumination techniques are needed to properly extract needed features [5].

Primarily, alpr system has two objectives: first, it must find the license plate location, and second, it needs to recognize the alphanumeric characters on the plate [6].

In the first objective there is a presence of problematic localization of plate number. This problem may be resolved through the implementation of algorithms, that can help detecting the rectangular area of the number plate in original image. A plate number can easily be identified by humans since we have an idea that this is a "small plastic or metal plate attached to a vehicle for official identification purposes", but machines cannot. Because of this, there is a need to find an alternative definition of the number plate based on descriptors, which will be allow the machine to identify it [7]. 
On the other hand, machine printed optical character recognition (ocr) can be seen by researcher as a "solved problem.". Although researchers are challenged to conduct ocr on complex images like license plates images which include complicated backgrounds, degradation, low resolution and rotation [8].

Today, numerous advances have been made in the field to address such issues, yet only worked under restricted condition i.e. single detection, images as input, perfect camera angle, etc. There are also other studies applying multiple detection i.e. images as input but with straight camera angle and specified distance which in return recognizes only 2-3 maximum vehicle at once [9], from video input but focuses only on the recognition of over speeding buses [10], in real time but suffers to low quality videos and high blur factor [11] and some other studies that focus only on plate localization and doesn't include recognition of characters.

Thus the researchers extended the study into multiple license plate recognition among philippines license plate format from video sequences, this also focused on image de-blurring to reduce blurring effects cause by out-offocus camera, motion blur and camera angle that is a problem associated mostly in this study.

\section{2.related works}

Automatic license plate recognition, abbreviated as alpr, become and active topic this past few years. This technology made use of computer vision and recognition of characters using a special algorithm to recognize a license plate and the development of a model or machine to do this function. This machine is dependent to different tools like a good quality camera, frame grabber, software application that can help with image processing. The success of an alpr can be divided in different factors like the efficiency of capturing the object. This necessitate to measure the number of the licence plate correctly captured from the number of vehicles data. Accuracy in the interpretation of the captured license plate in another factor to consider, this is called "read accuracy". Note that there are several circumstances that highly affect the capture or read factors. Read factors include plate design factors, plate covers or other known obstructions including dent, dirt, or any noises from the original image), problematic image resolution (resulting from the distance of the plate to the extractor of the image). Poor lighting and low contrast can also be included which are the product of overexposure, reflection, shadows, or changes in weather condition. Another to take in consideration is the interval of the vehicles to the angle of the camera capturing the image [2]

the factors presented roughly divildes the process of automatic licence plate recognition into three stages: the first one is plate localization, second, character segmentation and the last one is character recognition. Every step is considered as independent modules that will perform it functions considering the different factors. The basic alpr system usually start with reading the input video that was captured by the camera and will then proceed to each stage mentioned.[12]

\subsection{Plate localization/plate detection (pd)}

This stage is the process of obtaining the license plate region out of an image. This includes basic image processing techniques with decision making based on deterministic threshold. The processing will be done not considering any advance knowledge on the plate size and location. Its entire image must be inspected and processed to determine the candidate region to be extracted. Different approaches has been done to accomplish this, one is the connected component based methods which use regions a bottom-up approach by grouping pixels and components into larger components until all are identified [4], another is the edge-based methods focus on high contrast between the text and the background [9], a method called texture based that made used of the distinct textural properties in order to distinguish the text blocks from the background [11], alternative methods like window filtering can be used to match the size of the number plate proves to be useful when the sfr curve is generated. In this method only the values within the window are being added [13], a research of w. Jia used mean shift algorithm for localization of license plate giving satisfactory result for license plates having a different colour from the body colour [14], a study using adaptive threshold were done to adjust to the energy content (i.e. to the brightness, y component) of the image, after thresholding the original image, the brightest level is considered to be the background in the resultant picture, while the rest of the levels constitute the environment, characters and the frame of the number plate [15], and median filter method which is a non-linear filter that replaces gray value of a pixel by the median of the gray values of its neighbours, to add to these methods are the use of complex algorithms such as vector quantization, fuzzy clustering and fuzzy logic.

\section{2.character segmentation (cs)}

Once the detection of candidate region was done from an input image, the next step is to segment the plate to be able to extract each digit/character in proper recognition. This is dependent on the design of the plate to be processed. It is a fact that plate shapes and sizes vary for each country and regions, same with the plate's background and foreground colors and are totally different in length and combination of digit and characters. Different techniques were used for each specific cases and these can be adopted, an example of these techniques are combination of vertical and horizontal projection to determined glyph location [13], and adaptive clustering 
technique that helps find the white spaces between columns of higher density of dark pixels [5]. Adaptive binarization technique, called sliding concentric windows, is based on the standard deviation of the columns and rows in order to correctly segment the characters which is needed to solve the problem of vertical projection [16]. In terms of fragmented, superimposed or connected characters problems, adaptive segmentation and extraction was proposed based on mathematical morphology combined with heuristics that determine the points of potential segmentation that can help solve this problem[17]. Template matching method makes full use of the width of characters in the license plate arrangement rule features, design plate template string, the character position is determined by template matching, although this method has a good character segmentation, but this method is very complex [8]. A technique called blob extraction, aimed at detecting regions in a digital image that has different properties, was based in character segmentation to analyse blobs, a connected set of pixels in binary image, and removing the noisy blobs then merge and split to segment characters, segmentation can also use pixel connectivity which is the connected component analysis wherein it scans and labels the pixels of a binarized image into components. Pixels were labelled with a value depending on the component to which it was assigned [18]. Hough transform is used to find imperfect instances like image noise, problems in plate frame, rivet, space mark, plate rotation and lighting inconsistency. This method uses information of intensity and avoids the absorption that will overcome the drawbacks of binarization. Segment analysis engine technique find out the corresponding license plate regions from the edge map images. The motivation behind this work is to uplift the accuracy and the efficiency of license plate localization and to overcome the degradation and failure of result due to polluted environment and huge variation in the features of the license plate like different size, colour and font of the license plate and to generate a better method to segment the characters of the license plate [13]. Prior knowledge about the license plate is used along with projection method in order to eliminate the problems when projection method is used alone and helps in deleting the wrong horizontal segmentation and combining the correct lines, so character segmentation using prior knowledge of license plate seems simple and fast [14]. Despite of these abilities, these do not take into considerations that sometimes there could be frames that are partially connected to the plate content.

\section{3.character recognition}

Every digit and/or character extracted, must be identified, and ocr is responsible for that. There are several methods to attain this with a good accuracy. Two approaches are popular amongst the different researches on license plate recognition these are template matching [17] and the use of ann as classifier [18]. Template matching is a method using, a series of slightly different templates of all glyphs kept in a database. Once an image is submitted for recognition, existing templates will be compared to the new image, and the best fit will determine its identity. It is dependent on the size of the database if it is enough to cover most glyph variations, and it must also implement an efficient algorithm to process large set of templates. On the other hand, in using ann classifier, the model must be trained to recognize all the different digits, characters and symbols that require identification, therefore training data is the key in this method. Several other approaches are also being used such as feature analysis which is an analysis of the characters to distinguish it.

\section{3.system design}

figure 1 shows the system architecture for multiple license plate recognition. The system will start by

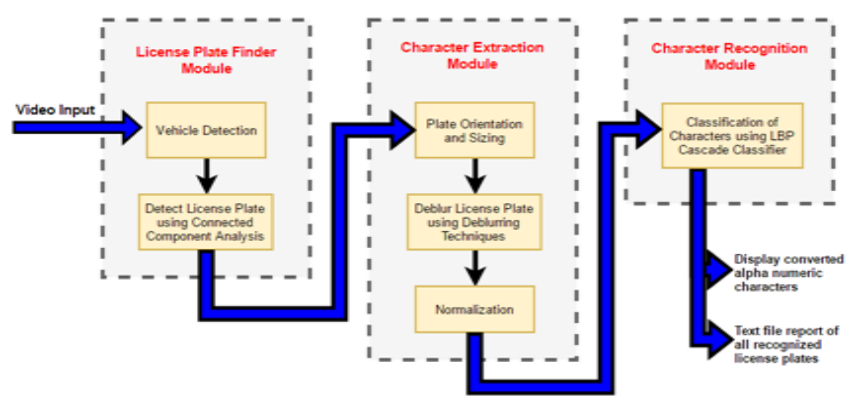

accepting an input in a form of video file.

Figure 1. System architecture

\section{1.license plate finder module}

First, the video input will be processed in the plate number finder to detect vehicle and extract it for the preparation of locating license plate. Next, the license plate will be detected and extracted from the detected vehicle, this will be processed using connected component analysis wherein it identifies connected component regions that share same set of intensity values, from this the license plate region will be determine. 


\section{2.character extraction module}

It will then proceed to the next module, the character extraction, here, the license plate will be oriented and sized to compensates for the skew of the plate and adjust the dimensions to the required size. Next, the license plate image will be de-blur using different deblurring techniques to eliminate the effects of blur. After that is the normalization process which change the range of pixel intensity values to enhance the contrast and brightness of the image, this is to improve the quality of the image.

\section{3.character recognition module}

When the image is enhanced and the characters are almost visible, the last unit will takes place which is the character recognition, in this unit the located and segmented characters in the license plate will be classify and converted into alphanumeric text, in order to do this lbp cascade classifier will be used as the main classifier of characters. The converted text will be displayed in the interface, and the system will also provide text report of all recognized license plate in the input video.

\section{4.methodology}

This study utilized an experimental research method. Generally, one or more independent variables are manipulated to determine their effect on a dependent variable. Moreover, experimental method of research discovers the influence of one or more factor upon a given condition or situation [19].

The researchers selected experimental type of research because the study intend to assess the relationship of independent variable to the dependent variable. This relationship between variables will help researchers prove the hypothesis. Different experiments and calculations will be performed to know what instances the application performs best.

The researchers gathered data along commonwealth avenue,quezon city, philippines, highway in a daylight weather and specific time of $9 \mathrm{am}-11 \mathrm{am}$ and $3 \mathrm{pm}-5 \mathrm{pm}$.

In taking videos of the samples, the researchers prepared a camera with a specs of at least $720 \mathrm{p}$ hd video recording (30fps) and sight camera of 8 megapixel. The camera is placed above the ground that formed an angle of elevation from the vehicle to the camera, so in achieve this, the video recording takes place in the footbridge, specifically near up-ayala techno hub in commonwealth avenue. Just one side of the road were captured wherein rear part of the vehicle is visible.

A total of 30 recordings with a length of 3 minutes each is the data gathered. 20 of this served as the training data and the remaining 10 were the testing data. In terms of training data, each license plate snap shots from the videos are cropped out and saved as an image. On the other hand, for experimentation, before trials begin, videos were processed wherein ground truth data are extracted, this served as the basis of measurements for recognition.

All videos were tested and statistically evaluated then the results were recorded accordingly. Finally, these data were organized, analyzed and summarized on such a way that it answers the problem of the study.

The researchers made used of precision, recall and f-measure to determine the degree of closeness and completeness of the results [20] that have been obtained in terms of detecting the plate number and root mean square (rms) to determine the recognition rate of license plate characters.

To derive the result needed for the study, the output of the system was classified using confusion matrix defined as follows:

Table 1. Confusion matrix for plate detection

\begin{tabular}{|c|c|c|c|}
\hline & \multicolumn{3}{|c|}{ Identified by the System } \\
\hline \multirow{3}{*}{$\varangle$} & & License plate & Not license plate \\
\cline { 2 - 4 } & License plate & True Positive (TP) & False Negative (FN) \\
\cline { 2 - 4 } & Not license plate & False Positive (FP) & True Negative (TN) \\
\hline
\end{tabular}

True positive (tp) - license plate was detected as license plate

False positive (fp) - it was detected as license plate but is not

True negative (tn) - it is not a license plate and detected as not license plate 
False negative (fn) - license plate was detected as not license plate

\section{5.discussion of the results}

The researchers have conducted this study to create a multiple recognition of license plates on video sequences as well as to incorporate image deblurring and analyze its capabilities to improve the recognition of characters in the license plates while still being applicable to multiple detection. Two modules are tested which is the plate detection which evaluated in terms of precision, recall, f-measure and for character recognition is in terms of root mean square (rms) or error value to find its accuracy.

The results are summarized as follows:

Table 2. Summary of findings in terms of plate detection

\begin{tabular}{|l|c|c|c|c|c|c|}
\hline & TP & FN & FP & PRECISION & RECALL & F-MEASURE \\
\hline Without deblurring & 597 & 61 & 110 & 84.33 & 90.72 & 87.32 \\
\hline With deblurring & 597 & 61 & 110 & 84.33 & 90.72 & 87.32 \\
\hline
\end{tabular}

The results shows that for both systems without deblurring and with deblurring has the same findings in terms of plate detection because this module did not undergo the process of image deblurring thus the result has no effect so the precision $(84.33 \%)$, recall $(90.72 \%)$ and f-measure $(87.32 \%)$ stays the same for the two conditions. It is also observed that there are more successful license plate to be detected but the detected license plates can be less relevant, the possible reason for this is, in most cases there are improper segmentation of license plate to the other details in the background. Some cases includes, first is the vehicle having the same color with its license plate. Since connected component analysis group same pixel intensity into blocks, some of the resulted blocks, include other details in backgrounds as a license plate resulting into larger components not acceptable for normal license plate size, and second there are different changes in intensity value due to its contrast, resulting in not properly grouping license plate as one component, thus detecting some rectangular objects as license plate, when it is really not.

Table 3. Summary of findings in terms of character recognition

\begin{tabular}{|c|c|c|}
\hline & RMS & ACCURACY \\
\hline Without deblurring & 51.75 & 48.25 \\
\hline With deblurring & 37.33 & 66.26 \\
\hline
\end{tabular}

The computation for accuracy in character recognition was done by getting first the rms or error rate and then subtracting it to 100 to obtain the accuracy, as we can see the accuracy of character recognition with deblurring which is $66.26 \%$ is greater than the accuracy of without deblurring which is $48.25 \%$. As observed, the reason of having such results is because of less training data for some characters, it is said that the data are just randomly selected which means that some characters aren't in

Table 4. Summary of comparison between the system with and without deblurring

\begin{tabular}{|c|c|c|}
\hline & Without Debluriing & With Debluring \\
\hline Mean & 48.25 & 62.66 \\
\hline Standard Deviation & 4.07 & 3.96 \\
\hline Degree of Freedom & \multicolumn{2}{|c|}{18} \\
\hline Critical Value & \multicolumn{2}{|c|}{3.92} \\
\hline T-Value & 8.03 \\
\hline
\end{tabular}


The license plate or only few of the characters are retrieved from the set of license plates. Also there are characters in the license plates that are partially blocked by objects or dirt which makes it harder for the classifier to distinguish the characters.

In terms with significant difference between the developed systems with deblurring and the system without deblurring, it shows that the calculated t-value which is 8.03 is greater than the critical value of 3.92 given that the degree of freedom is 18 and the p-value is $<0.001$, this states that there is a significant difference between the two.

\section{6.conclusions and recommendations}

After experimentations and data analysis were done, the researchers have reached the following conclusions and generalizations: the developed system is capable of recognizing license plate from video sequences and can do multiple plate detection, it recognizes multiple license plate via video sequences and is able to perform better with the incorporation of image deblurring, the character recognition module was greatly affected by the number of training data. There is a significant difference between the result of the system with image deblurring and without image deblurring in recognizing multiple license plates.

The researchers believed that the study could be improved more so the following recommendations are given for the future researchers: utilize other machine learning algorithms for characters in the license plate that will be able to learn the construction of characters even if it is partially blocked by some objects, apply edge-based methods on segmenting license plates to reduce the effects of high contrast between license plate and background, and test the system on other weather or lighting conditions that might affect its detection or recognition. 